\title{
Sarcopenia, systemic immune-inflammation index and all-cause mortality in middle-aged and older people with COPD and asthma: a population-based study
}

\author{
Elizabeth Benz $\mathbb{( \mathbb { D }}^{1,2}$, Sara R.A. Wijnant $\mathbb{D}^{1,3,4}$, Katerina Trajanoska $\mathbb{C}^{1,2}$, Johnmary T. Arinze $\mathbb{1}^{3,5}$, \\ Emmely W. de Roos $\mathbb{D}^{1,3}$, Maria de Ridder $\mathbb{C}^{5}$, Ross Williams $\mathbb{C}^{5}$, Frank van Rooij $\mathbb{D}^{1}$,

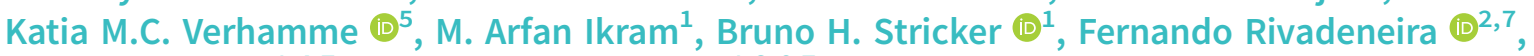 \\ Lies Lahousse (1) ${ }^{1,4,7}$ and Guy G. Brusselle (D) ${ }^{1,3,6,7}$
}

\begin{abstract}
${ }^{1}$ Dept of Epidemiology, Erasmus MC - University Medical Center Rotterdam, Rotterdam, The Netherlands. ${ }^{2}$ Dept of Internal Medicine, Erasmus MC - University Medical Center Rotterdam, Rotterdam, The Netherlands. ${ }^{3}$ Dept of Respiratory Medicine, Ghent University Hospital, Ghent, Belgium. ${ }^{4}$ Dept of Bioanalysis, Faculty of Pharmaceutical Sciences, Ghent University, Ghent, Belgium. ${ }^{5}$ Dept of Medical Informatics, Erasmus MC - University Medical Center Rotterdam, Rotterdam, The Netherlands. ${ }^{6}$ Dept of Respiratory Medicine, Erasmus MC - University Medical Center Rotterdam, Rotterdam, The Netherlands. ${ }^{7}$ These authors contributed equally.
\end{abstract}

Corresponding author: Guy G. Brusselle (guy.brusselle@uzgent.be)
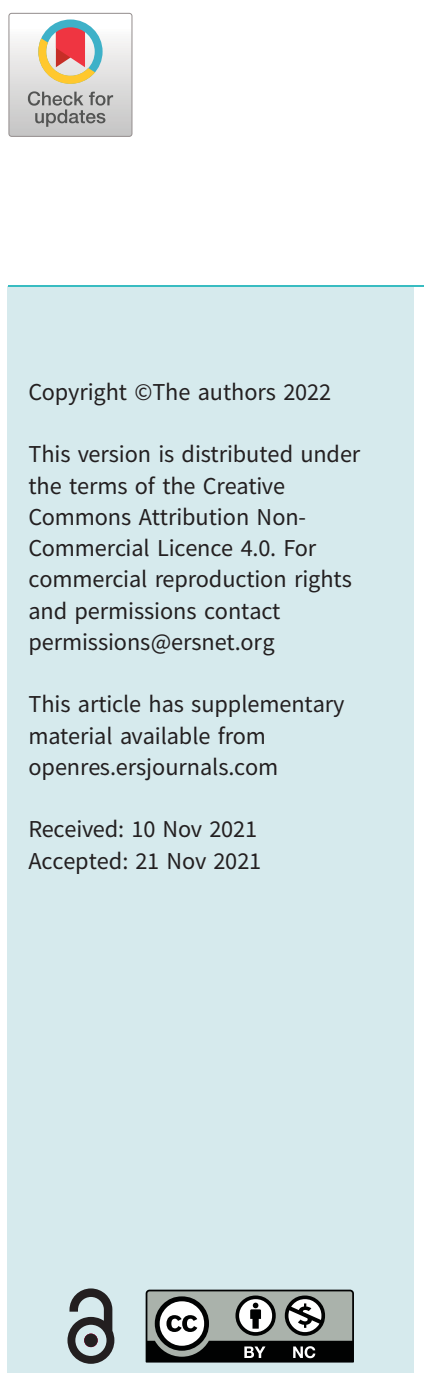

Shareable abstract (@ERSpublications)

Sarcopenia and COPD increased the risk of all-cause mortality in middle-aged and older populations. Additionally, compared to lower SII levels, higher SII levels increased mortality risk in people with and without sarcopenia and COPD. https://bit.ly/3d659m2

Cite this article as: Benz E, Wijnant SRA, Trajanoska K, et al. Sarcopenia, systemic immuneinflammation index and all-cause mortality in middle-aged and older people with COPD and asthma: a population-based study. ERJ Open Res 2022; 8: 00628-2021 [DOI: 10.1183/23120541.00628-2021].

\section{Abstract}

Background Increasing evidence suggests that sarcopenia and a higher systemic immune-inflammation index (SII) are linked with morbidity in patients with COPD. However, whether these two conditions contribute to all-cause mortality in middle-aged and older patients with COPD or asthma is unclear. Therefore, we investigated the association between sarcopenia, SII, COPD or asthma and all-cause mortality in a large-scale population-based setting.

Methods Between 2009 and 2014, 4482 participants (aged >55 years; 57.3\% female) from the populationbased Rotterdam Study were included. COPD and asthma patients were diagnosed clinically and based on spirometry. Six study groups were defined according to the presence or absence of COPD or asthma and sarcopenia. Cox regression models were used to assess all-cause mortality in the study groups, adjusted for sex, age, body mass index, SII, smoking, oral corticosteroid use and comorbidities. In addition, all participants were categorised into sex-specific quartiles of SII, and mortality in these groups was compared.

Results Over a median follow-up of 6.1 years (interquartile range 5.0-7.2 years), 466 (10.4\%) persons died. Independent of the presence of sarcopenia, participants with COPD had a higher risk of all-cause mortality (hazard ratio (HR) 2.13, 95\% CI 1.46-3.12 and HR 1.70, 95\% CI 1.32-2.18 for those with and without sarcopenia, respectively). Compared to lower SII levels, higher SII levels increased mortality risk even in people without sarcopenia, COPD or asthma.

Conclusion Middle-aged and older people with COPD, higher SII levels or sarcopenia had an independently increased mortality risk. Our study suggests prognostic usefulness of routinely evaluating sarcopenia and SII in older people with COPD or asthma.

\section{Introduction}

COPD and asthma are heterogeneous chronic airway conditions in the older population and are highly prevalent worldwide [1]. COPD is the third leading cause of death [2], and older patients with asthma also experience a higher risk of morbidity and mortality [3]. A subgroup of patients with COPD and asthma shares a key feature, systemic inflammation, linked with lung function decline and the pathophysiology of 
musculoskeletal comorbidities [4-6]. Within the comorbidities framework of these chronic airway diseases, sarcopenia, characterised by the loss of muscle mass and decreased muscle function [7], is a common extrapulmonary condition, particularly in middle-aged and older patients [8]. Depending on diagnostic method, definition criteria and the population setting, a large range of prevalence of sarcopenia in subjects with COPD has been reported (between $8 \%$ and 63\%) [9]; in older asthmatics from the general population, nearly $5 \%$ have reported sarcopenia [8].

In patients with COPD, systemic inflammatory biomarkers have been linked with sarcopenia [5, 10]; however, this effect has not been fully explored in asthmatic patients. Systemic inflammation encompasses a complex network of interactions, including immune-related cells such as neutrophils and lymphocytes [11]. A recent development is that the systemic immune-inflammation index (SII) appears to be a novel marker for chronic inflammation, reflecting increased absolute blood counts of neutrophils and platelets, and decreased counts of blood lymphocytes [12, 13]. Higher SII levels have been associated with worse prognosis and increased mortality in patients with cancer or cardiovascular diseases [14, 15]. Moreover, a higher neutrophil-to-lymphocyte ratio is a marker of systemic inflammation in COPD exacerbations [11, 16]. Subsequently, there is an increased interest in its usefulness in chronic conditions in the respiratory field [17].

Patients with sarcopenia, high SII levels, COPD and, to a lesser extent, asthma have been independently associated with increased risk of all-cause mortality compared to those without sarcopenia, low SII levels, COPD or asthma [11, 18-20]. However, our understanding of whether the combination of COPD or asthma and sarcopenia is related to the risk of all-cause mortality in older people remains limited. Such data might contribute to guiding early interventions as primary prevention.

We hypothesised that middle-aged and older people with COPD or asthma and sarcopenia would have increased risk of all-cause mortality, particularly those with higher SII levels. Therefore, our main objective was to investigate whether these combined conditions of COPD or asthma and sarcopenia increase mortality risk in a middle-aged and older population. Our secondary objective was to explore whether SII levels modulate the effect of sarcopenia and chronic airway diseases on all-cause mortality.

\section{Methods}

Study design and participants

This study was conducted within the Rotterdam Study, an ongoing population-based cohort that started in 1989, involving $\sim 15000$ participants aged $\geqslant 45$ years, investigating determinants of several chronic diseases [21, 22]. Every 4-5 years, participants from the Ommoord district (in Rotterdam, the Netherlands) undergo follow-up examinations at the research centre (i.e. spirometry) [22].

Our study included all participants with valid data on spirometry, blood sample and sarcopenia, who visited the research centre between 2009 and 2014 (supplementary figure S1). Other relevant data were retrieved from general practitioners' medical records and prescription data from pharmacies serving the Ommoord area. The Rotterdam Study was approved by the medical ethics committee of the Erasmus Medical Center, Rotterdam (registration number MEC 02.1015), and by the Dutch Ministry of Health, Welfare and Sport (Population Screening Act WBO, license number 1071272-159521-PG). All participants provided written informed consent to participate in the Rotterdam Study and obtain their information from treating physicians.

\section{Exposure definition and measurements \\ COPD and asthma}

Trained paramedic personnel performed pre-bronchodilator spirometry using a MasterScreen PFT Pro spirometer (CareFusion, Houten, the Netherlands) according to the American Thoracic Society and the European Respiratory Society guidelines [23]. Predicted values of forced expiratory volume in $1 \mathrm{~s}\left(\mathrm{FEV}_{1}\right)$ and forced vital capacity (FVC) were calculated employing the reference equations obtained from the Global Lung Function Initiative, which take into account age, sex, height and ethnicity [24]. COPD cases were defined by spirometry data $\left(\mathrm{FEV}_{1} / \mathrm{FVC}\right.$ ratio $\left.<0.7\right)$ and medical records. Asthma was based on physician-diagnosis, as evidenced in the medical records [25]. Asthma-COPD overlap (ACO) cases were identified as asthmatic participants who met the obstructive spirometry definition of COPD. We excluded participants with other lung diseases, ACO, no data on lung function or uninterpretable spirometry. If there was no record for diagnosis of chronic airway diseases or normal spirometry (FEV $/ \mathrm{FVC} \geqslant 0.7$ and $\mathrm{FEV}_{1}$ $\geqslant 80 \%$ ) at the time of sarcopenia measurement, participants were assumed to be without COPD, asthma, ACO or other lung diseases. 
Sarcopenia

We defined sarcopenia according to the updated European Working Group of Sarcopenia in Older People (EWGSOP2) criteria (supplementary table S1).

Maximum handgrip strength was obtained from the nondominant hand after three trials using a hydraulic dynamometer (Fabrication Enterprises, White Plains, New York, NY, USA). Appendicular lean mass was measured by dual-energy X-ray absorptiometry (DXA) (iDXA total body-beam densitometer; GE Lunar Corp, Madison, WI, USA). Each scan was analysed using enCORE software V13.6. The sum of the lean mass from the upper and lower limbs is referred as appendicular lean mass (ALM). Appendicular lean mass index (ASMI) was defined as ALM divided by the height squared $\left(\mathrm{m}^{2}\right)$. Probable sarcopenia was defined as having a low handgrip strength ( $<27 \mathrm{~kg}$ for males and $<16 \mathrm{~kg}$ for females) [7], and was confirmed as having a low ASMI $\left(<7.0 \mathrm{~kg} \cdot \mathrm{m}^{-2}\right.$ for males and $<5.5 \mathrm{~kg} \cdot \mathrm{m}^{-2}$ for females) [7]. We merged all participants with probable sarcopenia (low handgrip strength) and confirmed sarcopenia (low handgrip strength and low ASMI) as sarcopenia definition.

\section{Study groups}

All participants were categorised in six study groups according to the presence/absence of COPD or asthma and sarcopenia: 1) no COPD, asthma or sarcopenia (reference); 2) COPD without sarcopenia; 3) COPD with sarcopenia; 4) asthma without sarcopenia; 5) asthma with sarcopenia; and 6) sarcopenia only (supplementary table S2).

\section{Systemic immune-inflammation index}

Fasting blood samples were collected at the study centre. Full blood counts were performed on a Coulter Ac.T diff2 Hematology Analyzer (Beckman Coulter, San Diego, CA, USA). The SII was calculated from the platelets $\left(\times 10^{9}\right.$ cells $\left.\cdot \mathrm{L}^{-1}\right)$, granulocytes, as a proxy for neutrophils $\left(\times 10^{9}\right.$ cells $\left.\cdot \mathrm{L}^{-1}\right)$, and lymphocytes $\left(\times 10^{9}\right.$ cells $\left.\cdot \mathrm{L}^{-1}\right)$, using the formula $\mathrm{SII}=$ platelets $\times$ neutrophils/lymphocytes [12]. Sex-specific quartiles of SII were defined for all participants.

\section{Outcome and covariates measurements}

Information on vital status was obtained from general practitioners and municipal records. Follow-up time started at the date of DXA scan assessment and ended at the date of death or the end of the study (30 May 2018), whichever came first.

For each participant in this study, we retrieved information on the following covariates: age groups $(<70$ and $\geqslant 70$ years); sex; smoking status (never, past and current); smoking pack-years (dichotomised at 20 pack-years: $<20$ pack-years and $\geqslant 20$ pack-years); body mass index (BMI) $\left(\mathrm{kg} \cdot \mathrm{m}^{-2}\right)$; oral corticosteroid use (Anatomical Therapeutic Chemical code: H02; never: no prescriptions in the complete medical history, past: at least one prescription $>3$ months before DXA date, current: at least one prescription within the 3 months preceding the DXA date); and physical activity using a validated adapted version of the Longitudinal Aging Study Amsterdam Physical Activity Questionnaire, expressed in metabolic equivalent of task per week.

Moreover, the comorbidity count was defined as none, one or two or more comorbidities, and a fourth category was used for participants with an unknown number of comorbidities (at least one missing). The comorbidities included were type 2 diabetes (fasting plasma glucose level $\geqslant 7 \mathrm{mmol} \cdot \mathrm{L}^{-1}$, or a nonfasting plasma glucose level $\geqslant 11.1 \mathrm{mmol} \cdot \mathrm{L}^{-1}$ or the use of blood glucose-lowering medication); hypertension (systolic blood pressure $\geqslant 140 \mathrm{mmHg}$ or diastolic blood pressure $\geqslant 90 \mathrm{mmHg}$ or use of antihypertensive medication); coronary heart disease (myocardial infarction, coronary artery bypass grafting or percutaneous coronary intervention); osteoporosis (T-score $\leqslant-2.5$, according to World Health Organization criteria); depression (using the validated Center for Epidemiologic Studies Depression Scale; scores $\geqslant 16$ were considered as major depressive symptoms). Participants with diagnoses of cancer were excluded.

\section{Statistical analysis}

First, we described baseline characteristics for all participants according to the six study groups. Differences in continuous variables between the study groups were tested using one-way ANOVA, and only when the p-value was $<0.05$, post hoc pairwise comparisons were performed. For skewed continuous variables, the Dunn test was applied. Categorical variables were compared using the Chi-squared test (all p-values are based on nonmissing values). 
Second, time to death (survival probability) was described using Kaplan-Meier curves and log-rank or Peto-Peto tests for participants: 1) with and without COPD or asthma; 2) with and without sarcopenia; 3) with different SII quartiles; and 4) in the six study groups.

Third, to evaluate the differences in all-cause mortality between study groups, we performed four Cox regression models, including an increasing number of covariates: model 0 was unadjusted. In model 1 , we adjusted for age groups ( $<70$ and $\geqslant 70$ years) and sex. In model 2 we additionally adjusted for SII quartiles. In model 3, we accounted for previous confounders in model 2, adding BMI, smoking pack-years and oral corticosteroid use. In model 4 , we additionally adjusted for the number of comorbidities. Likelihood ratio tests were performed to assess interaction terms between study groups and age groups, sex and SII quartiles into model 4. In addition, we analysed the association between lower versus higher SII quartiles and all-cause mortality in the six study groups. Cox regression models with progressive adjustment for the aforementioned confounders were fitted. We confirmed the assumptions of proportional hazards by statistical evaluation of Schoenfeld residual plots, inspecting for symmetry over time and the p-values.

Lastly, several sensitivity analyses were done. 1) As the vast majority of the sarcopenia cases were participants with low handgrip strength (probable sarcopenia), we repeated the analysis by excluding the cases of low handgrip strength and low ASMI (confirmed sarcopenia). 2) Since physical activity has been associated previously with mortality risk, we adjusted for physical activity. 3) We adjusted for lung function ( $\mathrm{FEV}_{1} \%$ predicted). 4) Finally, we explored the association between SII quartiles and all-cause mortality in all population and two separate subsets: 1) all groups without sarcopenia and 2) all groups with sarcopenia. Effect estimates were plotted as hazard ratios (HR) for time to death, alongside their corresponding 95\% confidence intervals. For $11.3 \%(n=508)$ of missing data for physical activity, we used multiple imputation technique (20 imputations with 30 interactions for each imputation), with the mice package of the $\mathrm{R}$ software. Statistical analysis was performed using $\mathrm{R}$ version 3.5.1 (Foundation for Statistical Computing, Vienna, Austria). A p-value $<0.05$ was considered to indicate significance in all analyses.

\section{Results}

Baseline characteristics of the study population

Among 7162 participants of the Rotterdam Study, 5013 (70.0\%) had complete data on spirometry, sarcopenia and SII between 2009 and 2014. Of these, we excluded 509 participants diagnosed with cancer and 22 with ACO, resulting in 4482 (63.0\%) participants for the analysis. According to the six study groups, 3074 (68.6\%) participants had no COPD nor asthma, nor sarcopenia (reference); 589 (13.1\%) had COPD without sarcopenia; 92 (2.1\%) had COPD with sarcopenia; 329 (7.3\%) had asthma without sarcopenia; 62 (1.4\%) had asthma with sarcopenia; and 336 (7.5\%) had sarcopenia only (figure 1).

Compared to the reference group, participants of all sarcopenic categories were on average older and more likely to have three or more comorbidities. In addition, they had higher SII levels, except for the group with asthma and sarcopenia (table 1).

\section{Mortality in the study population}

The median (IQR) follow-up time was 6.1 (5.0-7.2) years. Over this period, 466 (10.4\%) participants died. Of those, 211 (45.3\%) had no COPD, asthma or sarcopenia; 99 (21.2\%) had COPD without sarcopenia; 36 (7.7\%) had COPD with sarcopenia; 21 (4.5\%) had asthma without sarcopenia; 34 (7.3\%) had asthma with sarcopenia; and 86 (18.5\%) had sarcopenia only (supplementary table S2).

The survival probability was worse in subjects with COPD compared to those without COPD and without asthma (figure 2a; log-rank $\mathrm{p}<0.001$ ) and in subjects with sarcopenia compared to those without sarcopenia (figure $2 b$; log-rank $\mathrm{p}<0.001$ ). Moreover, there were significant differences in mortality between SII quartiles (figure 2c; log-rank $\mathrm{p}<0.001$ ), and participants with higher values of SII (quartile 4) had worse survival probability than those with lower values of SII (quartile 1).

All-cause mortality between study groups

According to the study groups, Kaplan-Meier analyses for survival probability showed significant differences (figure 3; Peto-Peto $\mathrm{p}<0.001$ ).

Table 2 describes the association of the study groups with all-cause mortality. Compared to the reference group, in the fully adjusted model (model 4), COPD with and without sarcopenia had a higher risk of all-cause mortality (HR 2.13, 95\% CI 1.46-3.12 and HR 1.70, 95\% CI 1.32-2.18), as well as participants with sarcopenia only (HR 2.09, 95\% CI 1.61-2.70). Subjects with asthma and sarcopenia were at increased 


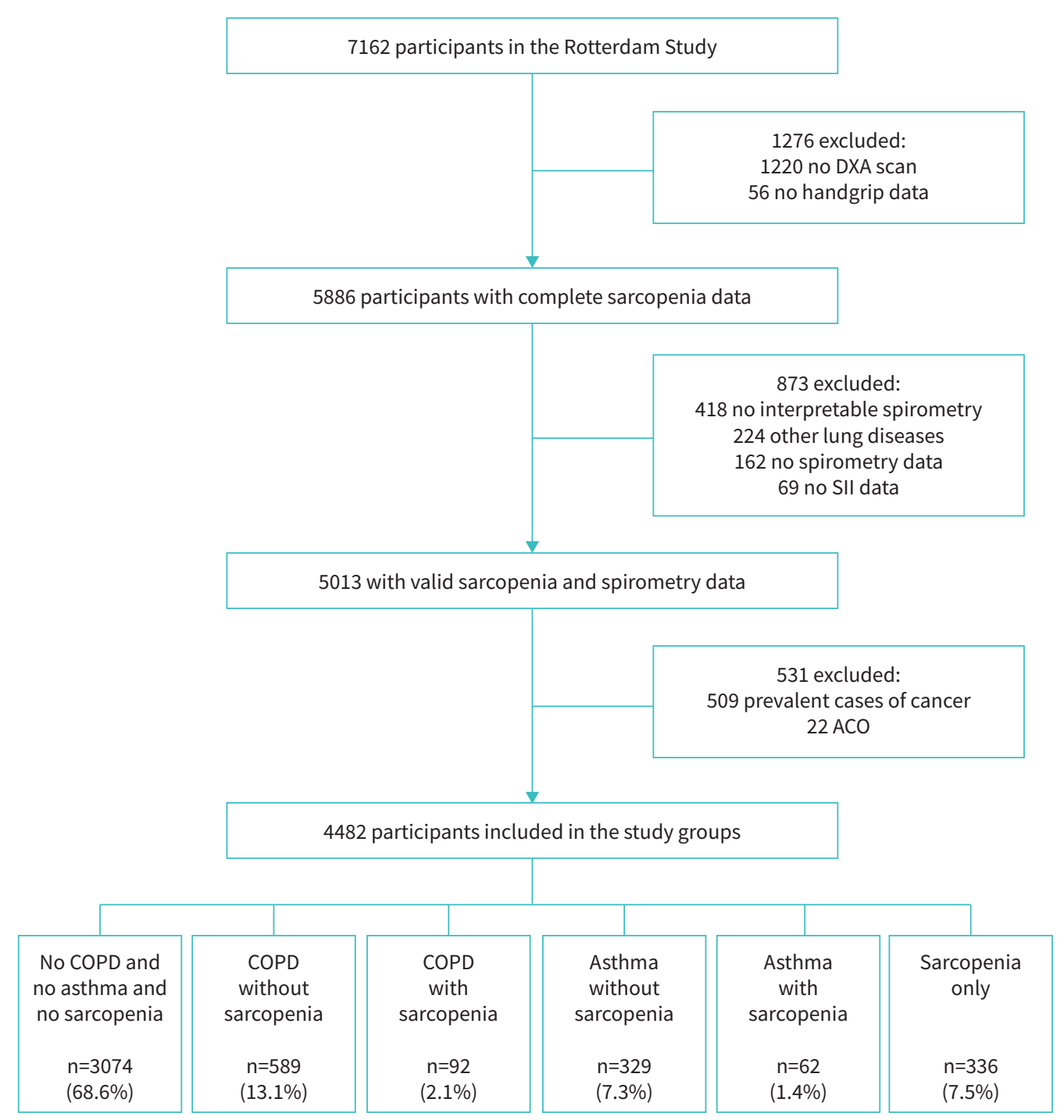

FIGURE 1 Flowchart for the participants included in the six study groups. DXA: dual-energy X-ray absorptiometry; SII: systemic immune-inflammation index; ACO: asthma-COPD overlap.

risk of all-cause mortality, but adjusting for the number of comorbidities attenuated this association (HR 1.75, 95\% CI 0.99-3.10) (table 2, figure 4). We found no evidence for age, sex and SII interactions with study groups for all-cause mortality and no deviations from the proportional hazards assumption.

\section{All-cause mortality according to levels of systemic immune-inflammation index}

Regarding the association of SII levels and all-cause mortality, in the study groups, subjects with higher levels of SII had an increased risk of mortality than those with lower levels of SII, particularly subjects with sarcopenia only, COPD without sarcopenia and the reference group (model 2; table 3). This association is attenuated by the number of comorbidities (model 3; table 3).

\section{Sensitivity analysis}

When the analysis was repeated by excluding participants with confirmed sarcopenia ( $n=69)$ (low handgrip strength with normal ASMI), the estimator did not materially change in all study groups (supplementary figures S2, S3 and supplementary table S3). Additional adjustment for physical activity did not affect the effect estimate in all study groups (supplementary table S4), whereas $\mathrm{FEV}_{1}$ (\%) attenuated the effect in COPD participants (supplementary table S4). When we focused on the association of SII and all-cause mortality in all participants $(\mathrm{n}=4482)$, subjects with higher levels of SII had a significant increase in mortality risk than those with lower levels of SII (HR 1.74, 95\% CI 1.33-2.28) (supplementary figure 
TABLE 1 Baseline characteristics in the six study groups.

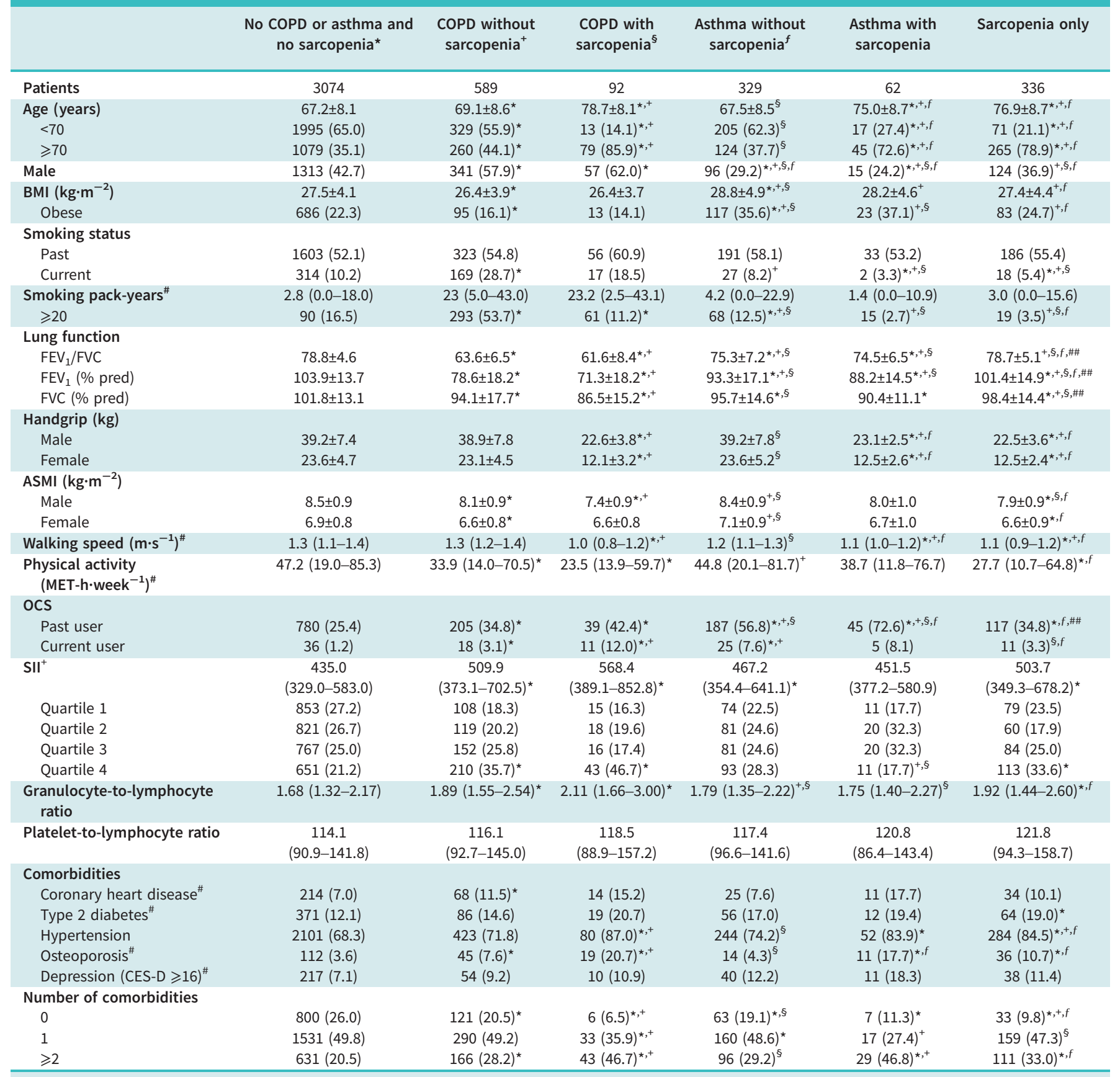

Data are presented as $\mathrm{n}$, mean $\pm \mathrm{SD}, \mathrm{n}(\%)$ or median (interquartile range). BMI: body mass index; $\mathrm{FEV}_{1}$ : forced expiratory volume in $1 \mathrm{~s}$; FVC: forced vital capacity; ASMI: appendicular skeletal muscle mass index; MET: metabolic-equivalent of task; OCS: oral corticosteroids; SII: systemic immune-inflammation index; CES-D: Center for Epidemiologic Studies Depression Scale. \#: missing values per variable: smoking pack-years n=7 $(0.2 \%)$; walking speed $n=1226(27.4 \%)$; physical activity $n=508(11.3 \%)$; comorbidities (coronary heart disease $n=49(1.1 \%)$, type 2 diabetes $n=79$ $(1.8 \%)$, hypertension $n=1(0.0 \%)$, osteoporosis $n=95(2.1 \%)$, depression $n=19(0.4 \%)$ ); number of comorbidities $n=186(4.1 \%)$ (original data without imputations); ${ }^{+}$: males quartile (Q) $1<341.2$, Q2 $>341$ and $<451.3$, Q3 $>451.3$ and $<608.0$, Q4 $>608.0$; females Q1 $<337.3$, Q2 $>337.3$ and $<451.5$, Q3 $>451.5$ and $<617.5$, Q4 >617.5. *: significant p-value $(<0.05)$ compared to reference group; ${ }^{+, ~} \$, f$, \#\#: significant p-value $(<0.05)$ between groups.

S4a). Similarly, in both subsets of participants, i.e. participants without sarcopenia, COPD or asthma $(n=3074)$, and participants with sarcopenia and COPD or asthma $(n=1408)$, subjects with higher levels of SII had a significantly increased mortality risk compared to those with lower levels of SII (HR 1.50, 95\% CI 1.02-2.21 and HR 1.87, 95\% CI 1.27-2.75, respectively) (supplementary figure S4b,c). These associations were independent of age groups, sex, BMI, pack-years of smoking, oral corticosteroid use and the number of comorbidities. 

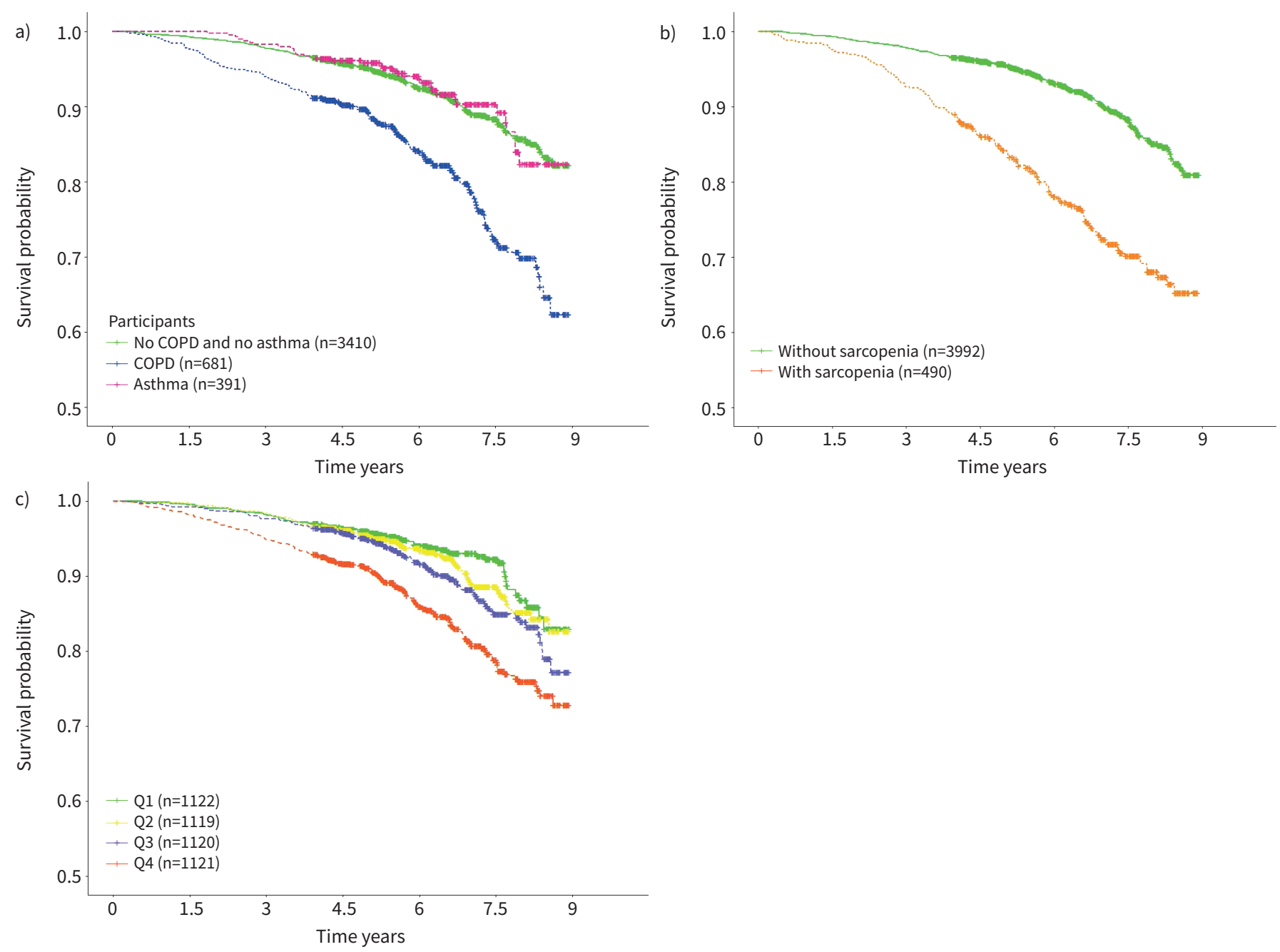

FIGURE 2 Kaplan-Meier curves for the survival time in participants a) with or without COPD or asthma; b) with or without sarcopenia; and c) according to quartiles of systemic immune-inflammation index (SII). Log-rank $p<0.001$ in a), b) and c).

\section{Discussion}

One of the main results of this population-based study was that middle-aged and older people with COPD (with and without sarcopenia) and sarcopenia only have a significantly higher risk of all-cause mortality than people without COPD, asthma or sarcopenia. In addition, we observed that subjects with higher levels of SII had an increased mortality risk compared to those with lower SII levels. Our results indicate that sarcopenia and high levels of SII are important risk factors for mortality risk in people with and without COPD.

In line with our results, previous studies have reported a significant effect of sarcopenia on mortality in COPD patients [26-30]. Borda et al. [31] reported that sarcopenic COPD patients had a higher risk of all-cause mortality after 3 years of follow-up than those without COPD and sarcopenia. Our results expand these findings from BoRDA et al. in a larger population (encompassing people with COPD or asthma) over a more extended follow-up period.

On one hand, our results shown that high SII levels increase mortality risk even in people without sarcopenia, COPD or asthma; however, asthma does not increase the risk of all-cause mortality, except for asthmatic participants with sarcopenia, by which mainly the number of comorbidities seems to attenuate the risk. On the other hand, two previous studies from the Rotterdam Study found that high SII levels were associated with risk of cancer and dementia [14, 32]. Importantly, two recent studies have reported that high SII levels were associated with increases all-cause mortality risk in general population [33, 34]. 


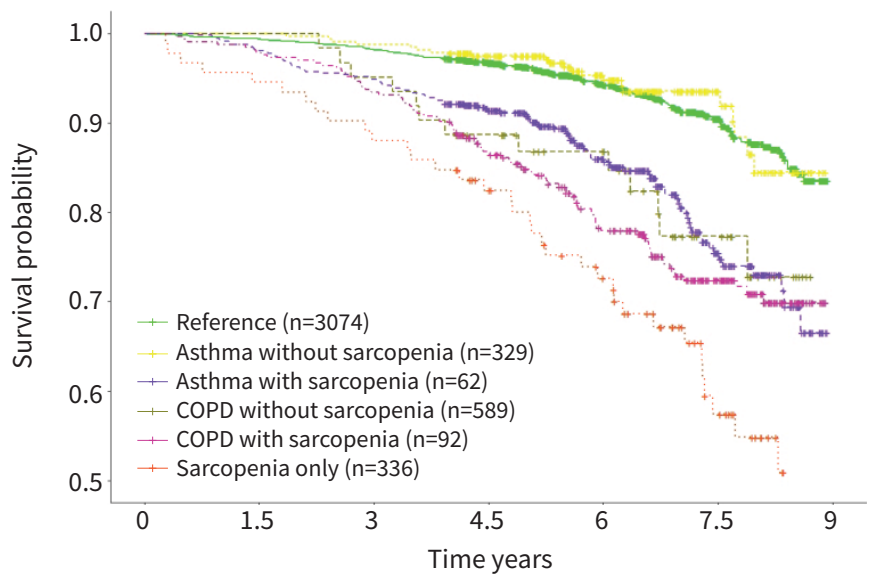

FIGURE 3 Kaplan-Meier estimator curve for survival time in all the participants according to the combination of presence/absence of COPD or asthma and sarcopenia status. Reference: no COPD, asthma nor sarcopenia. Peto-Peto test $p<0.001$

It is pivotal to examine how these biomarkers (i.e. SII) change during the pre-clinical phase of sarcopenia in older patients with chronic airway diseases. One aspect of immune senescence includes increased systemic inflammation, producing different cytokines (T-lymphocytes) linked with pro-inflammatory effects. For instance, elevated levels of tumour necrosis factor- $\alpha$ for a prolonged time are associated with muscle protein degradation at older ages [35, 36]. Moreover, neutrophils release free radicals during the immune system's activation, and muscle cells regulate this response by liberating nitric oxide [35, 37]. It may accumulate damaged protein during ageing due to lifelong exposure to free radicals, slowing muscle protein turnover [35, 38]. Clinical interest is increasing in the use of these haemogram indices, which might predict exacerbation or mortality in COPD. Although SII has been less studied [39], our study showed that levels of SII were increased in COPD and all-sarcopenic groups. Therefore, SII might be a useful index for further investigation and for clinical practice, because it is an additional inflammatory maker to complement the understanding of systemic inflammation pathways. Besides the clinical and population-based studies performed separately for sarcopenia and systemic immune-inflammatory index, further studies on chronic airway diseases are warranted to validate our results.

Strengths of this study include its population-based setting, robust clinical assessment of COPD and asthma (diagnosed by physicians with detailed medical records), high-quality criteria for the diagnosis of sarcopenia based on the EWGSOP2 statements (i.e. DXA scan and handgrip strength) and precise calculation regarding SII (blood sample). Nevertheless, this study has potential limitations that should be taken into account when interpreting these results. First, we had low numbers of events in the combined

TABLE 2 Association between the study groups and the risk of all-cause mortality

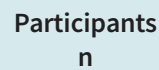

n

3070

No COPD, no asthma, no

sarcopenia

Asthma without sarcopenia

Asthma with sarcopenia

COPD without sarcopenia

COPD with sarcopenia

Sarcopenia only

329
62
589
92
333

\section{Unadjusted \\ model HR $(95 \% \mathrm{CI})$}

Model 1 HR $(95 \% \mathrm{Cl})$
Model 2

HR $(95 \% \mathrm{Cl})$
Model $3^{\#}$ HR $(95 \% \mathrm{Cl})$
Model 4 HR $(95 \% \mathrm{CI})$

Bold values indicate statistical significance $(p<0.05$ ). Model 1 : adjusted for sex and age ( $<70$ years and $\geqslant 70$ years); model 2 : model 1 adjusted for systemic immune-inflammation index (quartiles); model 3: model 2 adjusted for body mass index $\left(\mathrm{kg} \cdot \mathrm{m}^{-2}\right)$, smoking pack-years $(<20$ and $\geqslant 20$ pack-years) and oral corticosteroid use (no use, past and current use); model 4: model 3 adjusted for the number of comorbidities. Reference (Ref.): no COPD, asthma or sarcopenia. HR: hazard ratio. ": missing values: smoking pack-years $n=7$ (0.2\%); number of comorbidities $n=186$ (4.1\%). 


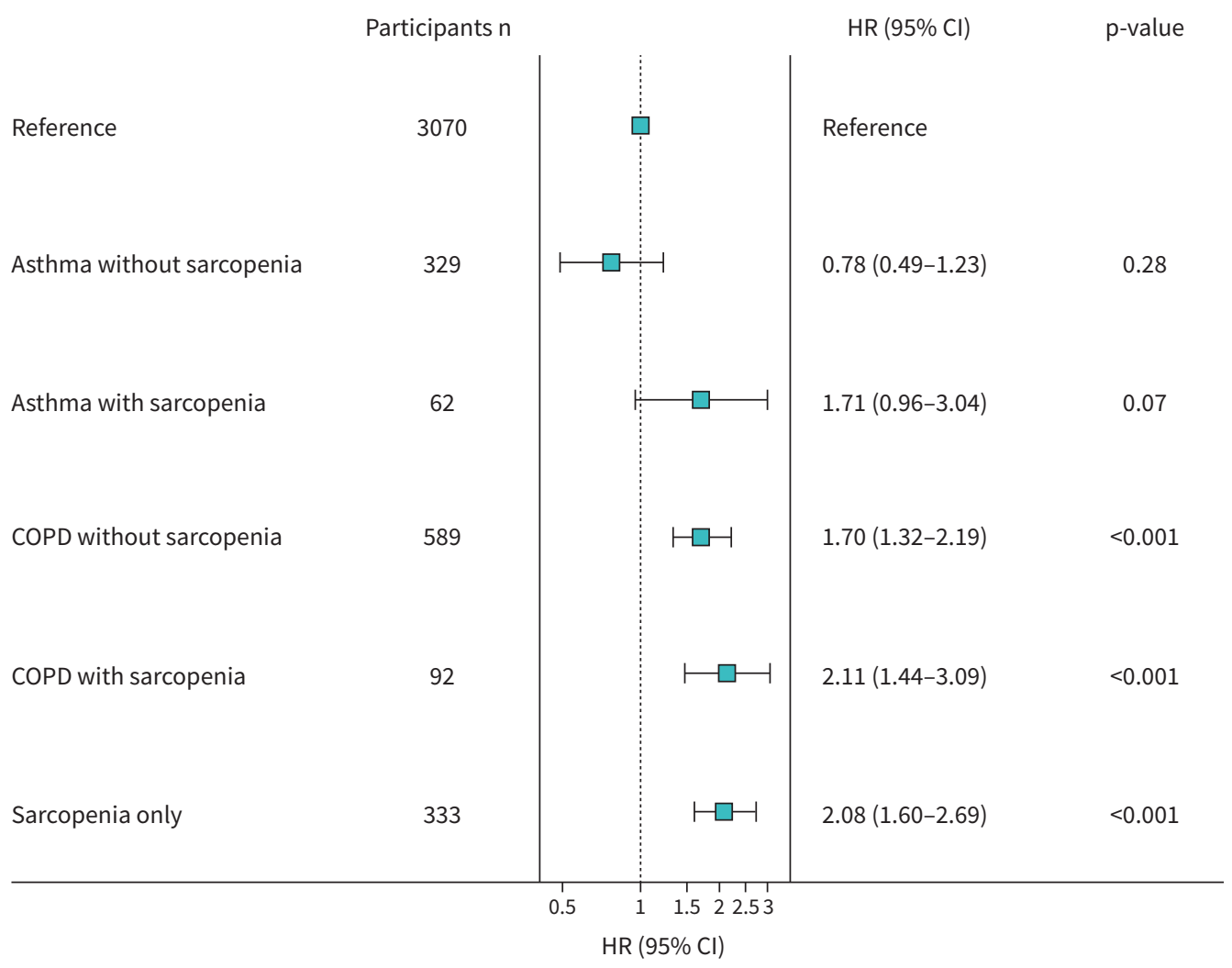

FIGURE 4 Forest plot of the association between the study groups and all-cause mortality. Data presented as adjusted hazard ratio (HR) with 95\% confidence intervals. Participants with no COPD, no asthma and no sarcopenia were used as the reference group. Sarcopenia includes both probable and confirmed sarcopenia according to the European Working Group of Sarcopenia in Older People definition. All analyses were adjusted for age, sex, systemic immune-inflammation index quartiles, smoking pack-years, body mass index, oral corticosteroid use and the number of comorbidities (model 4).

TABLE 3 Association between study groups and the risk of all-cause mortality according to the level of systemic immune-inflammation index (SII)

\section{Participants n Quartile of SII Unadjusted model HR $(95 \% \mathrm{Cl})$}

\begin{tabular}{lcccccc}
\hline Ref. & 3074 & Low SII & Ref. & Ref. & Ref. & Ref. \\
& 211 & High SII & $1.46(1.11-1.91)$ & $1.38(1.05-1.82)$ & $1.33(1.01-1.75)$ & $1.31(0.99-1.73)$ \\
Asthma without sarcopenia & 329 & Low SII & Ref. & Ref. & Ref. & Ref. \\
& 21 & High SII & $1.72(0.70-4.26)$ & $1.52(0.61-3.80$ & $1.44(0.57-3.65)$ & $1.49(0.59-3.78)$ \\
Asthma with sarcopenia & 62 & Low SII & Ref. & Ref. & Ref. & Ref. \\
& 13 & High SII & $1.26(0.42-3.76)$ & $1.32(0.44-3.95)$ & $1.21(0.36-4.00)$ & $0.93(0.26-3.28)$ \\
COPD without sarcopenia & 589 & Low SII & Ref. & Ref. & Ref. & Ref. \\
& 99 & High SII & $1.73(1.11-2.70)$ & $1.66(1.07-2.59)$ & $1.59(1.02-2.48)$ & $1.53(0.97-2.40)$ \\
COPD with sarcopenia & 92 & Low SII & Ref. & Ref. & Ref. & Ref. \\
& 36 & High SII & $1.58(0.76-3.29)$ & $1.58(0.76-3.29)$ & $1.20(0.55-2.60)$ & $1.27(0.57-2.79)$ \\
Sarcopenia only & 336 & Low SII & Ref. & Ref. & Ref. & Ref. \\
& 86 & High SII & $1.58(1.00-2.49)$ & $1.60(1.01-2.53)$ & $1.66(1.05-2.63)$ & $1.56(0.98-2.49)$ \\
\hline
\end{tabular}

Bold values indicate statistical significance $(p<0.05)$. Model 1 : adjusted for sex and age ( $<70$ years and $\geqslant 70$ years); model 2 : model 1 adjusted for body mass index $\left(\mathrm{kg} \cdot \mathrm{m}^{-2}\right)$, smoking pack-years ( $<20$ and $\geqslant 20$ pack-years) and oral corticosteroid use (no use, past and current use); model 3: model 2 adjusted for the number of comorbidities. Reference (Ref.): no COPD, asthma or sarcopenia. HR: hazard ratio; low SII: sex-specific SII quartiles (1 and 2); high SII: sex-specific SII quartiles (3 and 4). \#: missing values: smoking pack-years $n=7$ (0.2\%); number of comorbidities $n=186$ (4.1\%). 
groups of sarcopenia with COPD or asthma; therefore, it was not always possible to conclude whether SII levels modulate their mortality risk. However, when we analysed two subsets of participants separately: one subset with more-sick people ( $\mathrm{n}=1408$, sarcopenia and COPD or asthma) and another subset with less-sick people ( $n=3074$, no sarcopenia and no COPD or asthma), we found that high SII levels had more significant mortality risk than the low SII levels. Second, we did not consider the specific cause of death analyses; however, previous studies have reported that sarcopenia was associated with higher respiratory mortality risk [19]. Third, out of all participants with sarcopenia $(n=490)$, most of them had probable sarcopenia $(n=421)$, and few had confirmed sarcopenia $(n=69)$. When we performed a sensitivity analysis by including only cases with probable sarcopenia (low handgrip strength), the estimator (HR) remained similar in all study groups, which might mean that having only a low handgrip strength increases mortality risk in older people. Nevertheless, more research, which includes only cases with confirmed sarcopenia (low handgrip strength and low ASMI), needs to be performed to quantify the effect on survival, particularly in the older COPD population. Although we believe these results are generalisable to Dutch middle-aged and older people, the applicability of our result to other populations is anticipated, but cannot be definitively addressed by our study. In addition, we observed a high percentage of obesity in all study groups; thus, further research might be focused on this phenotype: sarcopenic-obesity combined with SII levels [40].

In conclusion, our study has shown that COPD, sarcopenia and high SII levels are significantly associated with increased risk of all-cause mortality in middle-aged and older people. These results also reported that the presence of sarcopenia and high levels of SII are independent risk factors for all-cause mortality in middle-aged and older people with or without chronic airway diseases (COPD or asthma). In addition, our analysis suggests benefits in terms of prognostic information when systematically evaluating these independent factors such as sarcopenia and SII index in middle-aged and older patients, particularly those with COPD.

Acknowledgements: The authors are grateful for the dedication, commitment and contribution of the citizens, general practitioners and pharmacists of the Ommoord district who took part in the Rotterdam Study.

Provenance: Submitted article, peer reviewed.

Conflict of interest: E. Benz has nothing to disclose. S.R.A. Wijnant reports grants from GlaxoSmithKline outside the submitted work. K. Trajanoska has nothing to disclose. J.T. Arinze reports a doctoral research grant from Merck Sharp \& Dohme, paid to their institution, outside the submitted work. E.W. de Roos has nothing to disclose. M. de Ridder has nothing to disclose. R. Williams has nothing to disclose. F. van Rooij has nothing to disclose. K.M.C. Verhamme reports that they work for a research department that receives or has received unconditional grants from Yamanouchi, Pfizer/Boehringer Ingelheim, Novartis, GSK, UCB, Amgen and Chiesi, none of which are related to the content of this paper. M.A. Ikram has nothing to disclose. B.H. Stricker has nothing to disclose. F. Rivadeneira has nothing to disclose. L. Lahousse reports financial support for the current manuscript from the Fund for Scientific Research Flanders (grant 3G037618), and payment or honoraria for lectures, presentations, speakers' bureaus, manuscript writing or educational events from Institute for Continuing Study of Pharmacists, outside the submitted work. G.G. Brusselle reports receiving fees for advisory boards or lectures from AstraZeneca, Boehringer Ingelheim, Chiesi, GlaxoSmithKline, Novartis, Sanofi and Teva, outside the submitted work.

Support statement: The Rotterdam Study was supported by the Erasmus Medical Center, Erasmus University Rotterdam, the Netherlands Organisation for Scientific Research (NWO), the Netherlands Organisation for Health Research and Development (ZonMw), the Netherlands Heart Foundation, the Research Institute for Diseases in the Elderly (RIDE), the Netherlands Genomics Initiative (NGI), the Ministry of Education, Culture and Science, the Ministry of Health Welfare and Sports, the European Commission (DG XII), and the Municipality of Rotterdam; and financial support by the Fund for Scientific Research Flanders (grant 3G037618). The funding sources had no involvement in the collection, analysis, writing, interpretation or in the decision to submit the paper for publication. Funding information for this article has been deposited with the Crossref Funder Registry.

\section{References}

1 Soriano JB, Abajobir AA, Abate $\mathrm{KH}$, et al. Global, regional, and national deaths, prevalence, disability-adjusted life years, and years lived with disability for chronic obstructive pulmonary disease and asthma, 1990-2015: a systematic analysis for the Global Burden of Disease Study 2015. Lancet Respir Med 2017; 5: 691-706.

2 World Health Organization. Chronic Obstructive Pulmonary Disease (COPD). www.who.int/news-room/ fact-sheets/detail/chronic-obstructive-pulmonary-disease-(copd)/ 2020. Date last updated: 26 February 2021. 
3 Busse PJ, McDonald VM, Wisnivesky JP, et al. Asthma across the ages: adults. J Allergy Clin Immunol Pract 2020; 8: 1828-1838.

4 Agusti A, Faner R, Donaldson G, et al. Chronic Airway Diseases Early Stratification (CADSET): a new ERS Clinical Research Collaboration. Eur Respir J 2019; 53: 1900217.

5 Byun MK, Cho EN, Chang J, et al. Sarcopenia correlates with systemic inflammation in COPD. Int J Chron Obstruct Pulmon Dis 2017; 12: 669-675.

6 Global Initiative for Asthma. Global Strategy for Asthma Management and Prevention. Available from: www.ginasthma.org/ 2020. Date last updated: 1 August 2020.

7 Cruz-Jentoft AJ, Bahat G, Bauer J, et al. Sarcopenia: revised European consensus on definition and diagnosis. Age Ageing 2019; 48: 16-31.

8 Benz E, Trajanoska K, Schoufour JD, et al. Sarcopenia in older people with chronic airway diseases: the Rotterdam study. ERJ Open Res 2021; 7: 00522-2020.

9 Benz E, Trajanoska K, Lahousse L, et al. Sarcopenia in COPD: a systematic review and meta-analysis. Eur Respir Rev 2019; 28: 190049.

10 Joppa P, Tkacova R, Franssen FME, et al. Sarcopenic obesity, functional outcomes, and systemic inflammation in patients with chronic obstructive pulmonary disease. J Am Med Dir Assoc 2016; 17: 712-718.

11 Paliogiannis P, Fois AG, Sotgia S, et al. Neutrophil to lymphocyte ratio and clinical outcomes in COPD: recent evidence and future perspectives. Eur Respir Rev 2018; 27: 170113.

12 Hu B, Yang X-R, Xu Y, et al. Systemic immune-inflammation index predicts prognosis of patients after curative resection for hepatocellular carcinoma. Clin Cancer Res 2014; 20: 6212-6222.

13 Zhong J-H, Huang D-H, Chen Z-Y. Prognostic role of systemic immune-inflammation index in solid tumors: a systematic review and meta-analysis. Oncotarget 2017; 8: 75381-75388.

14 Fest J, Ruiter R, Mulder M, et al. The systemic immune-inflammation index is associated with an increased risk of incident cancer - a population-based cohort study. Int J Cancer 2020; 146: 692-698.

15 Fani L, van der Willik KD, Bos D, et al. The association of innate and adaptive immunity, subclinical atherosclerosis, and cardiovascular disease in the Rotterdam Study: a prospective cohort study. PLoS Med 2020; 17: e1003115.

16 Lu FY, Chen R, Li N, et al. Neutrophil-to-lymphocyte ratio predicts clinical outcome of severe acute exacerbation of COPD in frequent exacerbators. Int J Chron Obstruct Pulmon Dis 2021; 16: 341-349.

17 Ruta VM, Man AM, Alexescu TG, et al. Neutrophil-to-lymphocyte ratio and systemic immune-inflammation index - biomarkers in interstitial lung disease. Medicina 2020; 56: 381.

18 Landi F, Cruz-Jentoft AJ, Liperoti R, et al. Sarcopenia and mortality risk in frail older persons aged 80 years and older: results from ilSIRENTE study. Age Ageing 2013; 42: 203-209.

19 Petermann-Rocha F, Yang S, Gray SR, et al. Sarcopenic obesity and its association with respiratory disease incidence and mortality. Clin Nutr 2020; 39: 3461-3466.

20 Engelkes M, de Ridder MAJ, Svensson E, et al. Multinational cohort study of mortality in patients with asthma and severe asthma. Respir Med 2020; 165: 105919.

21 Hofman A, Grobbee DE, de Jong PTVM, et al. Determinants of disease and disability in the elderly: the Rotterdam Elderly Study. Eur J Epidemiol 1991; 7: 403-422.

22 Ikram MA, Brusselle G, Ghanbari M, et al. Objectives, design and main findings until 2020 from the Rotterdam Study. Eur J Epidemiol 2020; 35: 483-517.

23 Celli BR, MacNee W, Agusti A, et al. Standards for the diagnosis and treatment of patients with COPD: a summary of the ATS/ERS position paper. Eur Respir J 2004; 23: 932-946.

24 Quanjer PH, Stanojevic S, Cole TJ, et al. Multi-ethnic reference values for spirometry for the 3-95-yr age range: the global lung function 2012 equations. Eur Respir J 2012; 40: 1324-1343.

25 de Roos EW, Lahousse L, Verhamme KMC, et al. Asthma and its comorbidities in middle-aged and older adults; the Rotterdam Study. Respir Med 2018; 139: 6-12.

26 Marquis $\mathrm{K}$, Debigaré $\mathrm{R}$, Lacasse $\mathrm{Y}$, et al. Midthigh muscle cross-sectional area is a better predictor of mortality than body mass index in patients with chronic obstructive pulmonary disease. Am J Respir Crit Care Med 2002; 166: 809-813.

27 Schols AMWJ, Broekhuizen R, Weling-Scheepers CA, et al. Body composition and mortality in chronic obstructive pulmonary disease. Am J Clin Nutr 2005; 82: 53-59.

28 Slinde F, Grönberg A, Engström C-P, et al. Body composition by bioelectrical impedance predicts mortality in chronic obstructive pulmonary disease patients. Respir Med 2005; 99: 1004-1009.

29 Tanimura K, Sato S, Fuseya Y, et al. Quantitative assessment of erector spinae muscles in patients with chronic obstructive pulmonary disease. Novel chest computed tomography-derived index for prognosis. Ann Am Thorac Soc. 2016; 13: 334-341.

30 Attaway $\mathrm{AH}$, Welch N, Hatipoğlu U, et al. Muscle loss contributes to higher morbidity and mortality in COPD: an analysis of national trends. Respirology 2021; 26: 62-71. 
Borda MG, Celis-Preciado CA, Pérez-Zepeda MU, et al. Sarcopenia en ancianos con antecedente de EPOC/ asma: resultados del estudio SABE-Bogotá. [Sarcopenia in the elderly with a history of COPD/asthma: results of the SABE-Bogotá study]. Rev Esp Geriatr Gerontol 2017; 52: 313-316.

32 van der Willik KD, Fani L, Rizopoulos D, et al. Balance between innate versus adaptive immune system and the risk of dementia: a population-based cohort study. J Neuroinflammation 2019; 16: 68.

33 Jin Z, Wu Q, Chen S, et al. The associations of two novel inflammation indexes, SII and SIRI with the risks for cardiovascular diseases and all-cause mortality: a ten-year follow-up study in 85,154 individuals. J Inflamm Res 2021; 14: 131-140.

34 Li H, Wu X, Bai Y, et al. Physical activity attenuates the associations of systemic immune-inflammation index with total and cause-specific mortality among middle-aged and older populations. Sci Rep 2021; 11: 12532.

35 Meng S-J, Yu L-J. Oxidative stress, molecular inflammation and sarcopenia. Int J Mol Sci 2010; 11: 1509-1526.

36 Mitchell WK, Williams J, Atherton P, et al. Sarcopenia, dynapenia, and the impact of advancing age on human skeletal muscle size and strength; a quantitative review. Front Physiol 2012; 3: 260.

37 Dalle S, Rossmeislova L, Koppo K. The role of inflammation in age-related sarcopenia. Front Physiol 2017; 8 : 1045.

38 Phillips SM, Paddon-Jones D, Layman DK. Optimizing adult protein intake during catabolic health conditions. Adv Nutr 2020; 11: S1058-S1069.

39 Erdogan T. Role of systemic immune-inflammation index in asthma and NSAID-exacerbated respiratory disease. Clin Respir J 2021; 15: 400-405.

40 Schoufour JD, Tieland M, Barazzoni R, et al. The relevance of diet, physical activity, exercise, and persuasive technology in the prevention and treatment of sarcopenic obesity in older adults. Front Nutr 2021; 8: 661449. 